\title{
The spacing effect in intentional and incidental free recall by children and adults: Limits on the automaticity hypothesis
}

\author{
Thomas C. Toppino, Melodie D. Fearnow-Kenney, \\ Marissa H. Kiepert, ANd Amanda C. Teremula \\ Villanova University, Villanova, Pennsylvania
}

\begin{abstract}
Preschoolers, elementary school children, and college students exhibited a spacing effect in the free recall of pictures when learning was intentional. When learning was incidental and a shallow processing task requiring little semantic processing was used during list presentation, young adults still exhibited a spacing effect, but children consistently failed to do so. Children, however, did manifest a spacing effect in incidental learning when an elaborate semantic processing task was used. These results limit the hypothesis that the spacing effect in free recall occurs automatically and constrain theoretical accounts of why the spacing between repetitions affects recall performance.
\end{abstract}

Repetition facilitates learning and memory. However, the benefit of repetition depends on the spacing between successive occurrences of the repeated information. When repetitions are spaced (i.e., separated in time or by the presentation of other events), subsequent retention is better than when repetitions are massed (i.e., occur in immediate succession). This phenomenon, which is known as the spacing effect, has uncommon generality. It has been obtained with a variety of different learning materials (e.g., Hintzman \& Rogers, 1973; Kraft \& Jenkins, 1981; Mammarella, Russo, \& Avons, 2002), with many different measures of performance (e.g., Challis, 1993; Melton, 1970; Underwood, Kapelak, \& Malmi, 1976), and with diverse populations ranging from young children (e.g., Toppino, 1991) to elderly adults (e.g., Balota, Duchek, \& Paullin, 1989).

Over the years, numerous theories have been offered to explain the spacing effect. They can be categorized with respect to two dimensions: the mechanism that is assumed to produce the mnemonic benefit of spacing, and whether the mechanism operates automatically (involuntarily) or strategically (voluntarily). In terms of the mechanism, most theorists have proposed either a deficient-processing or an encoding-variability mechanism (Hintzman, 1974). However, recent hypotheses often have included studyphase retrieval as an important mechanism.

According to deficient-processing theories, massing repetitions results in one occurrence being inadequately processed (e.g., insufficient attention or a low level of processing), which hinders subsequent memory. When repetitions are spaced, both occurrences of a repeated item are thought to be more fully or more adequately processed, leading to better retention.
According to encoding-variability theories, each occurrence of a spaced repetition is assumed to be encoded differently, whereas massed repetitions are thought to be encoded similarly. Differential or variable encoding is assumed to facilitate memory by enabling a greater number of potentially effective retrieval routes.

Another potential cause of the spacing effect is studyphase retrieval, the retrieval of an item's prior presentation when a subsequent repetition is presented for study (Braun \& Rubin, 1998; Thios \& D'Agostino, 1976). For example, Thios and D' Agostino proposed that study-phase retrieval may provide a form of retrieval practice that becomes more similar to retrieval on the final test - and, thus, more beneficial for final test performance - as the spacing between repetitions increases.

In recent years, research and theorizing on the spacing effect have proceeded more or less independently for free recall and for cued-memory tasks, such as recognition and frequency judgments, in which a unique cue is provided for each to-be-remembered item. This dichotomous approach was initiated by Greene (1989), who attributed the spacing effect to a voluntary deficient-processing mechanism in cued-memory tasks and to an automatic encodingvariability mechanism in free recall. He supported this hypothesis with experiments that indicated that the spacing effect in cued-memory tasks was obtained in intentionallearning conditions but not in incidental-learning conditions, whereas, in free recall, the effect was obtained with both intentional and incidental learning.

Subsequent research on cued-memory tasks has resulted in a refined deficient-processing theory, but has strongly supported an automatic-process, rather than voluntaryprocess, explanation of the spacing effect. Challis (1993)

T. C. Toppino, thomas.toppino@villanova.edu 
noted that Greene (1989) had used an orienting task in his incidental-learning conditions that did not require semantic processing. When Challis induced semantic processing of words during incidental learning, he obtained a spacing effect in cued memory tasks. Russo, and his colleagues (Mammarella, Avons, \& Russo, 2004; Mammarella, Russo, \& Avons, 2002; Russo \& Mammarella, 2002; Russo, Parkin, Taylor, \& Wilks, 1998) repeatedly obtained a spacing effect in cued-memory tasks following incidental learning, and their results have clarified the role played by the type of processing during learning. For example, Russo and Mammarella showed that, although structural/perceptual processing inhibits the spacing effect in cued memory of meaningful words, it produces a spacing effect when materials are not conducive to semantic processing (e.g., nonwords).

The dominant theoretical view that has emerged from this cued-memory research is an automatic deficientprocessing account based on short-term priming and transfer-appropriate processing (Challis, 1993; Russo et al., 1998). It is assumed that the first occurrence of a repeated item automatically primes its second occurrence, and that the degree of processing that an item receives on its second occurrence is inversely related to its level of activation from priming. The spacing effect occurs because activation from priming should be greatest for massed repetitions and should decline with increasing spacing. When retrieval conditions are conducive to conceptually driven or semantic processing (e.g., when semantically meaningful materials, such as words, are the to-be-remembered items), the effective mechanism is assumed to be semantic priming (Challis, 1993). However, when conceptually driven or semantic processing is precluded (e.g., by the use of materials that do not support semantic processing), the spacing effect is thought to be mediated by short-term perceptual priming (Mammarella et al., 2004; Russo et al., 1998).

In the case of free recall, there is less agreement about the mechanism underlying the spacing effect. Deficientprocessing and study-phase retrieval accounts continue to be viable and receive some support (e.g., Braun \& Rubin, 1998; Van Strien, Verkoeijen, Van der Meer, \& Franken, 2007). However, theorizing has tended to focus on the role of contextual information because it seems to distinguish between free recall and cued-memory tasks. That is, contextual cues are thought to play a major role in free recall, whereas their role is greatly reduced in cued-memory tasks in which target-specific cues are thought to dominate.

As part of his component-levels theory, Glenberg (1979) proposed a contextual variability account of the spacing effect in free recall. He assumed that contextual elements are automatically encoded with target information, and that contextual elements spontaneously change with the passage of time and other events. Thus, as the spacing between repetitions of an item increases, the number of different contextual cues encoded with the item on successive occasions will also increase. This should be sufficient to enhance free recall performance by increasing the likelihood of encountering previously encoded contextual cues at retrieval.

Greene (1989) suggested a variant of the contextual variability hypothesis that includes study-phase retrieval, and subsequent theorists have elaborated on this idea (Raaijmakers, 2003; Verkoeijen, Rikers, \& Schmidt, 2004). Although study-phase retrieval has been proposed as a mechanism that is sufficient for directly producing the spacing effect for reasons that were described earlier (Braun \& Rubin, 1998; Thios \& D'Agostino, 1976), it plays a secondary role when combined with contextual variability. Contextual variability continues to provide the primary explanation for why the spacing of repetitions benefits free recall, but in this version of the theory, it is assumed that contextual elements must be encoded with a single representation of the target item rather than being distributed across multiple representations. Study-phase retrieval of an item's first presentation at the time of its second occurrence presumably is required for new contextual elements to be added to the previously established representation. If study-phase retrieval were to fail, a new, independent representation would be formed, and the spacing effect would be eliminated.

Although there is no clear consensus about the cause of the spacing effect in free recall, it is widely held that the spacing effect is produced by a fundamental mechanism that operates automatically. This hypothesis has overwhelming support from at least four separate lines of converging evidence. First, a number of experiments have demonstrated that young adult learners exhibit a spacing effect in free recall under incidental-learning conditions that are thought to preclude the use of study strategies (e.g., Jensen \& Freund, 1981; Shaughnessy, 1976; Toppino \& Bloom, 2002; Verkoeijen, Rikers, \& Schmidt, 2005). Second, Russo et al. (1998) found that requiring young adults to engage in a tone detection task during list presentation impaired overall free recall relative to a focused-attention condition (no secondary task), but it did not alter the spacing effect. Third, fast presentation rates that should limit the time available for the execution of voluntary processes during learning reduce the overall level of recall by young adult learners, but they do not attenuate the spacing effect (e.g., Glanzer, 1969; Melton, 1970).

A fourth line of research supporting an automaticprocess explanation of the spacing effect has investigated free recall in children for whom memory development between the preschool years and adolescence is marked by increasingly effective use of mnemonic strategies (Schneider \& Bjorklund, 1998). Although very young children (preschoolers) show clear evidence of strategy use in some tasks, they do not spontaneously use effective strategies in list-learning tasks, such as those employed in research on the spacing effect (Pressley \& Hilden, 2006). Even when the precursors of strategies are observed in preschoolers, they typically have no effect on memory performance (e.g., Baker-Ward, Ornstein, \& Holden, 1984; Ornstein, Baker-Ward, \& Naus, 1988). However, by their early elementary school years, children spontaneously exhibit effective rehearsal strategies, and by around fourth grade, they also employ more sophisticated organizational strategies (Schneider \& Bjorklund, 1998).

Support for an automatic-process explanation of the spacing effect in free recall is provided by findings that preschool children exhibit a spacing effect (Rea \& Mo- 
digliani, 1987; Toppino, 1991, 1993), and that the spacing effect in free recall seems to remain invariant from preschool through late elementary school, a period that is characterized by striking increases in strategic sophistication (Rea \& Modigliani, 1987; Toppino, 1991; Toppino $\&$ DeMesquita, 1984). Perhaps the most compelling evidence for an automatic-process explanation of the spacing effect was provided by Toppino (1991, Experiment 2), who examined the spacing effect in 3-and 4-year-olds. Lists consisted of series of pictures of common objects that the children subsequently tried to free recall. The lists were presented for study at the rate of 1,2 , or $5 \mathrm{sec}$ per item. Some investigators (e.g., Stoff \& Eagle, 1971; Wilkinson $\&$ Koestler, 1983) have suggested that a 1-sec presentation rate may be fast enough to prevent adults from using a voluntary strategy. Given that voluntary strategies take time to execute, it seems inconceivable that children as young as 3 years of age could make voluntary decisions about how to process each item when a 1 -sec rate is used. Nevertheless, 3 - and 4-year-olds exhibited a spacing effect that was unattenuated, even at the fastest presentation rate.

The present investigation began with a study of the spacing effect in free recall by preschool and fifth-grade children under conditions of intentional and incidental learning. Naturally, we expected to obtain further support for the automaticity hypothesis. That is, both age groups were expected to exhibit a spacing effect in both intentional- and incidental-learning conditions. Although this may appear to have been an exercise in confirming the obvious, the sheer paucity of research on the spacing effect with children suggested to us that additional, converging evidence was desirable. We found that children in neither age group exhibited a spacing effect following incidental learning. This surprising finding was examined further in subsequent studies.

\section{EXPERIMENT 1}

\section{Method}

Participants and Design. The participants were 72 preschool children (mean age $=54.6$ months, range $=43-61$ months $)$ and 72 fifth-grade children (mean age $=131.8$ months, range $=123-148$ months) who attended preschools and elementary schools in suburban Philadelphia. The experiment used a $2 \times 3 \times 2$ mixed factorial design with two grade levels, three levels of repetition/spacing (repeated items, with either massed or spaced presentations, and oncepresented items), and two learning conditions. Repetition/spacing was varied within participants, and grade level and learning condition were between-participants variables. For each combination of grade level and learning condition, 3 children were assigned to each of 12 experimental lists, resulting in 36 children in each of the four between-participants conditions. The data from an additional 4 preschoolers and 1 fifth grader were discarded and replaced as a result of outside interruptions or inability to correctly perform the task.

Materials. In preparing experimental lists, we first created two list structures, each consisting of 21 positions, or slots. The first 3 and last 3 positions were reserved for once-presented primacy and recency buffers, respectively. In the middle portion of each list structure, 3 slots were reserved for experimental once-presented items and 12 for the presentation of repeated items. Of these 12, 6 were reserved for the two presentations of three massed-presentation (MP) items and 6 for the two occurrences of three spaced- or distributedpresentation (DP) items. The presentations of each MP item oc- curred in immediate succession, separated by the presentation of no other items. The occurrences of each DP item were separated by three presentations of other items. In order to control for extended recency effects, which are sometimes obtained in free recall (e.g., Underwood, 1969), the mean serial positions of the second occurrences of MP and DP items were equated with each other and with the mean serial positions of the once-presented items.

List items consisted of 15 black-and-white line drawings of common objects known to be familiar to young children. The pictures bore no strong perceptual, semantic, or phonemic (in the case of their labels) relationship to each other.

Final lists were created in the following manner: The first list involving each structure was constructed by randomly assigning pictures (i.e., once-presented items, MP items, DP items, primacy buffers, recency buffers) to experimental conditions, and then to slots reserved for that type of item in the structure. A Latin-square counterbalancing principle was then used to construct the remaining lists with each structure by rotating items among the three levels of repetition/spacing (once-presented, MP, DP). This ensured that, across lists, each picture served equally often in each repetition/ spacing condition.

As we will describe below, pictures were eventually presented on one side or the other of a CRT display. The positioning of the pictures (left or right side of the screen) was determined randomly with the following constraints: (1) Repetitions of a single picture had to occur on the same side of the screen; (2) across lists constructed from the same list structure, each type of item appeared on each side of the screen an equal number of times; and (3) no more than 5 successive pictures could occur on the same side of the screen.

Procedure. The children participated individually. They heard a standard set of instructions that explained the task as a "guessing game with pictures." The pictures were presented on a screen that was located directly in front of the children. The children were informed that some pictures would be seen only once, whereas others would appear twice. They were also told that some pictures would appear on the left side of the screen, whereas others would appear on the right. The pictures were presented at a rate of 1 picture every $6 \mathrm{sec}$, with each picture appearing on-screen for $2 \mathrm{sec}$, followed by a 4-sec blank interval. The children were instructed to make their guesses during the blank interval by pointing to the side of the screen on which they thought the next picture would appear. When the next picture did appear, the children could determine whether or not their guess was correct. The children were instructed not to say the name of the picture when it appeared.

A short practice list was presented at this point to ensure that the children understood the procedure. There was no recall test following the practice list. The children were then informed that they would now see a much longer list of pictures. Children in the incidentallearning conditions received no further instructions, whereas children in the intentional-learning conditions were informed of the recall test that would follow the experimental list, and they were encouraged to try very hard to remember the pictures. Following presentation of the experimental list, there was a 2-min period of free recall, during which the children tried to name the pictures they had seen.

\section{Results}

Figure 1 presents the mean percentage of correct free recall, as a function of learning condition (intentional vs. incidental), grade level, and repetition/spacing. The latter was actually a combination of two logically separable factors: repetition (once-presented vs. repeated) and the spacing between repetitions (MP vs. DP). Although we were primarily concerned with the effect of spacing, the effect of repetition was important for interpreting the former. A failure to find that spacing alters the effect of repetition (no spacing effect) would be much less interesting 


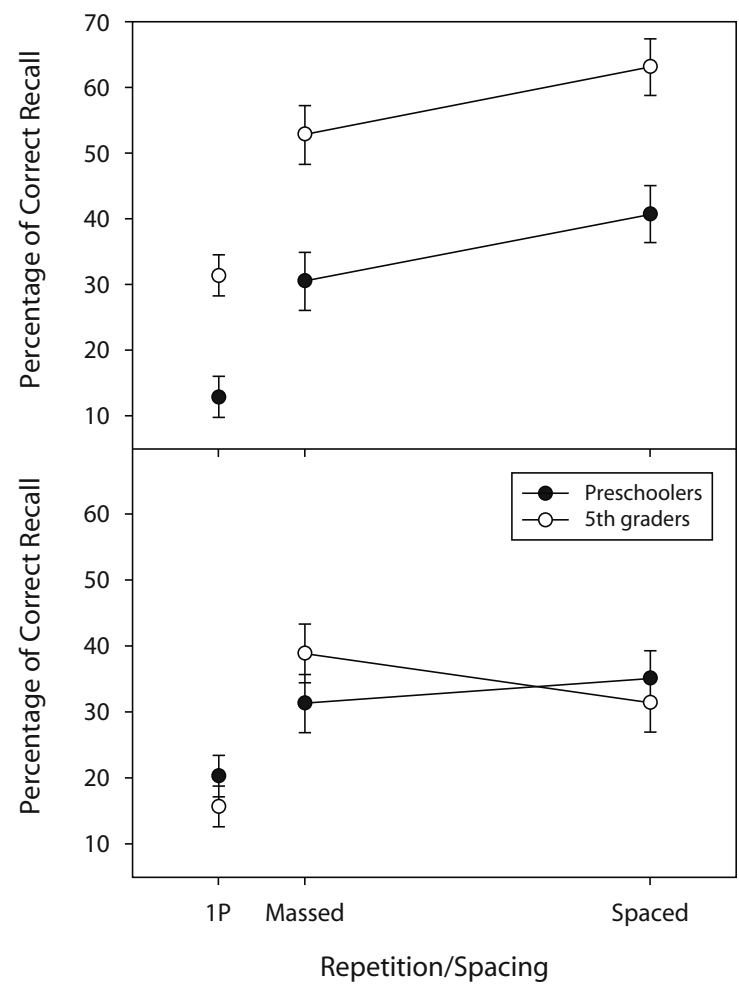

Figure 1. Mean percentage of correct free recall in Experiment 1 for fifth-grade children and preschool children in intentionallearning conditions (top panel) and incidental-learning conditions (bottom panel). 1P, once-presented items. Error bars represent $1 S E M$.

if the experimental conditions did not yield an effect of repetition in the first place. Therefore, the effects of spacing and of repetition were examined in separate three-way ANOVAs. Significance was set at $p=.05$ for all analyses in this study, unless otherwise stated.

The primary analysis examined the percentage of correct free recall, as a function of grade level, learning condition, and the spacing between repetitions (MP vs. DP) in a $2 \times 2 \times 2$ mixed ANOVA with repeated measures on the last factor. Significant main effects were obtained for grade level $\left[F(1,140)=14.86, M S_{\mathrm{e}}=713.05\right]$, indicating better recall for the fifth graders than for the preschoolers, and learning condition $\left[F(1,140)=16.04, M S_{\mathrm{e}}=\right.$ 713.05], indicating better recall for intentional- than for incidental-learning conditions. In addition, the fifth graders benefited more than did the preschoolers from intentional learning; this was reflected in the reliable grade level $\times$ learning condition interaction $[F(1,140)=10.51$, $\left.M S_{\mathrm{e}}=713.05\right]$.

The most important findings were that the main effect of spacing was not reliable $\left[F(1,140)=1.92, M S_{\mathrm{e}}=662.95\right]$, but that there was a significant interaction between spacing and learning condition $\left[F(1,140)=3.97, M S_{\mathrm{e}}=662.95\right]$. A spacing effect was obtained in the intentional-learning condition $[t(71)=2.55]$, with DP items being recalled better than MP items. However, no spacing effect was observed under incidental-learning conditions $[t(71)=$ $0.41]$. Finally, neither the grade level $\times$ spacing interaction nor the three-way interaction involving grade level, learning condition, and spacing approached significance [both $F_{\mathrm{s}}(1,140)<1.00, M S_{\mathrm{e}}=662.95$ ].

The second analysis was identical to the first, except that repetition (once-presented vs. twice-presented items) was substituted for spacing in a three-way ANOVA that also involved grade level and learning condition. For the purpose of this analysis, recall of twice-presented items was computed as the mean of the MP and DP conditions for each child. Most of the results were similar to those in the previous analysis and will not be repeated here. The most important finding was that repeated items ( $M=40.45)$ were recalled significantly better than oncepresented items $(M=20.01)\left[F(1,140)=76.09, M S_{\mathrm{e}}=\right.$ 395.13]. Although the interaction between repetition and learning condition approached significance, the repetition effect was reliable with both intentional learning $[t(71)=$ 7.00] and incidental learning $[t(71)=5.28]$. No other interaction approached significance $[F \mathrm{~s}(1,140)<1.25$, $\left.M S_{\mathrm{e}}=395.13\right]$.

\section{Discussion}

The results indicated that, in the intentional-learning conditions, the preschool children and the fifth graders exhibited a clear spacing effect. Taken alone, this finding is consistent with the hypothesis that the spacing effect is produced by an automatic mechanism, because the effect was obtained consistently across an age range in which sophistication in mnemonic strategies shows marked changes. However, the surprising fact that the spacing effect was eliminated in the incidental-learning conditions offers strong evidence against the automaticity hypothesis. It should be noted that the effect of repetition was not eliminated by incidental learning. Repeated items were recalled significantly better than once-presented items, regardless of whether learning was intentional or incidental. Only the effect of spacing between repetitions was influenced by the type of learning condition.

Previous developmental work on the spacing effect has, consistent with the intentional-learning results of the present experiment, demonstrated a spacing effect with very young children (e.g., Toppino, 1991, 1993) and developmental invariance from preschool through late elementary school (e.g., Rea \& Modigliani, 1987; Toppino \& DeMesquita, 1984). All of these studies employed intentionallearning conditions. In contrast, we are unaware of any prior investigation of the spacing effect with children that has used incidental learning. However, the fact that the children in the present experiment failed to produce a spacing effect in incidental learning is at odds with a number of studies involving adult participants (e.g., Jensen \& Freund, 1981; Shaughnessy, 1976; Toppino \& Bloom, 2002; Verkoeijen et al., 2005). Our findings also appear to be inconsistent with the wider body of research reviewed in the introduction, which converges strongly on the conclusion that the spacing effect in free recall is mediated by an involuntary, automatic mechanism in adult learners. 
The fact that the spacing effect was eliminated in the incidental-learning conditions was surprising; but even more surprising was the fact that this phenomenon was not limited to the youngest, least sophisticated children. Fifthgrade children possess a level of mnemonic and strategic sophistication that substantially overlaps that of adults; our results suggest, however, that incidental learning has a different influence on the spacing effect in these children than it does in adults. Therefore, in the remainder of this study, we focused on the spacing effect in late elementary school children, leaving further study of the spacing effect in very young children for a later time.

\section{EXPERIMENT 2}

The primary goal of Experiment 2 was to determine whether the surprising results of Experiment 1 were replicable. A secondary goal was to rule out the possibility that our procedures did not adequately control the spacing between repetitions. Our procedures included a 4-sec blank interval between successive presentations of list items, including between the first and second occurrences of massed-repetition items. It seemed at least possible that, in incidental-learning conditions with no mnemonic activity to bridge the gap, this interval may have been sufficient to convert nominal massed repetitions into functional spaced repetitions, thus eliminating the spacing effect. Therefore, we used fifth-grade children in an exact replication of Experiment 1 , except that a 2 -sec, rather than a 4 -sec, interval separated successive item presentations.

\section{Method}

Participants and Design. The participants were 48 fifth graders $($ mean age $=128.6$ months, range $=116-142)$, half of whom were randomly assigned to each learning condition (intentional vs. incidental) in a $2 \times 3$ (learning condition $\times$ repetition/spacing) mixed factorial design with the second factor varied within participants.

Materials and Procedure. Materials and procedures were identical to those used in Experiment 1, except that the blank interval separating successive item presentation was $2 \mathrm{sec}$.

\section{Results}

Figure 2 presents the mean percentage of correct free recall, as a function of learning condition and repetition/ spacing. The primary analysis was a $2 \times 2$ mixed ANOVA (learning condition $\times$ spacing) with repeated measures on the second factor. Intentional learning led to better recall than did incidental learning $[F(1,46)=13.90$, $\left.M S_{\mathrm{e}}=706.41\right]$. The main effect of spacing was not reliable $\left[F(1,46)=1.95, M S_{\mathrm{e}}=720.55\right]$, but the interaction between spacing and learning condition was significant $\left[F(1,46)=7.19, M S_{\mathrm{e}}=720.55\right]$. Children exhibited a spacing effect in the intentional-learning condition $[t(23)=2.64]$ but not in the incidental-learning condition $[t(23)=1.01]$.

The second analysis was identical to the first, except that repetition (once presented vs. twice presented) was substituted for the spacing variable. This analysis revealed a main effect of learning condition $[F(1,46)=17.98$, $\left.M S_{\mathrm{e}}=561.42\right]$ and a main effect of repetition $[F(1,46)=$ $\left.15.13, M S_{\mathrm{e}}=390.82\right]$, with repeated items $(M=39.89)$

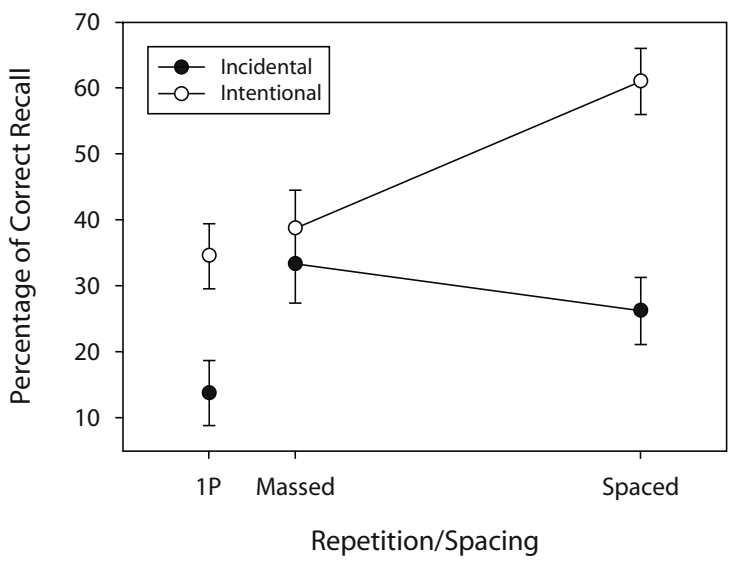

Figure 2. Mean percentage of correct free recall in Experiment 2 by fifth-grade children, as a function of intentional versus incidental learning, with a 2 -sec interval between items. 1P, oncepresented items. Error bars represent 1 SEM.

being recalled better than once-presented items $(M=$ 24.19). The interaction was not reliable $[F(1,46)<1.00]$.

\section{Discussion}

The results of this experiment clearly replicate the surprising outcome of Experiment 1. The children exhibited a spacing effect when learning was intentional, but not when it was incidental. Furthermore, the lack of a spacing effect was obtained under conditions in which twicepresented items were recalled better than once-presented items. Thus, the lack of a spacing effect cannot be trivialized as the product of conditions that eliminated all effects of repetition.

We replicated the children's performance in Experiment 1 , in spite of using a shorter (2-sec) interval between item presentations. Therefore, the children's failure to exhibit a spacing effect in incidental free recall cannot be easily attributed to an interstimulus interval that effectively turned all repeated items into spaced repetitions.

\section{EXPERIMENT 3}

The results of Experiments 1 and 2 are not consistent with studies in which adult participants exhibited a spacing effect in both intentional- and incidental-learning conditions (e.g., Toppino \& Bloom, 2002; Verkoeijen et al., 2005). This apparent discrepancy may reflect a developmentally related change in the way information is processed by children and by adults. However, adults have never been exposed to conditions comparable to those used in our Experiments 1 and 2. Perhaps they would perform similarly to the children if the conditions were the same. Therefore, in Experiment 3, we used college students to assess the spacing effect in free recall under conditions that were nearly identical to those used with the children in our previous experiments. The only difference was that the lists for the college students were longer. This was necessary in order to avoid ceiling effects. 


\section{Method}

Participants and Design. The participants were 96 college students, half of whom were randomly assigned to each of the learning conditions of a $2 \times 2$ mixed factorial design (learning condition $\times$ repetition/spacing) that was identical to the design of Experiment 2.

Materials and Procedure. Materials were the same as in Experiment 1 , except that the lists were lengthened for the adult participants. Four once-presented pictures were added to the beginning and the end of the list, so that there were seven primacy and seven recency buffers. In addition, one critical experimental item was added to each repetition/spacing condition for a total of four items representing each condition (once-presented, MP, DP). The instructions were modified slightly to make them more appropriate for adults. However, all other aspects of the lists and procedures were identical to those in Experiment 1.

\section{Results and Discussion}

Figure 3 presents the mean percentage of correct free recall, as a function of learning condition and repetition/ spacing. A $2 \times 2$ mixed design ANOVA indicated that recall was better in the intentional- than in the incidental-learning conditions $\left[F(1,94)=6.37, M S_{\mathrm{e}}=625.76\right]$. More important, there was a reliable main effect of spacing, in which recall was better for spaced than for massed repetitions $\left[F(1,94)=5.82, M S_{\mathrm{e}}=470.07\right] ;$ the interaction between spacing and learning condition did not approach significance $[F(1,94)<1.00]$. The second analysis involving repetition rather than spacing revealed only two main effects: Repeated items were recalled better than once-presented items $\left[F(1,94)=62.88, M S_{\mathrm{e}}=302.50\right]$, and intentional learning led to better performance than did incidental learning $\left[F(1,94)=11.23, M S_{\mathrm{e}}=428.16\right]$.

The fact that college students produced a spacing effect in both intentional- and incidental-learning conditions is consistent with previous results obtained with adults (e.g., Verkoeijen et al., 2005) and is consistent with the hypothesis that the spacing effect in free recall is produced by an automatic mechanism. The fact that children do not exhibit a spacing effect in essentially identical incidental-learning conditions, however, suggests that the automaticity of the

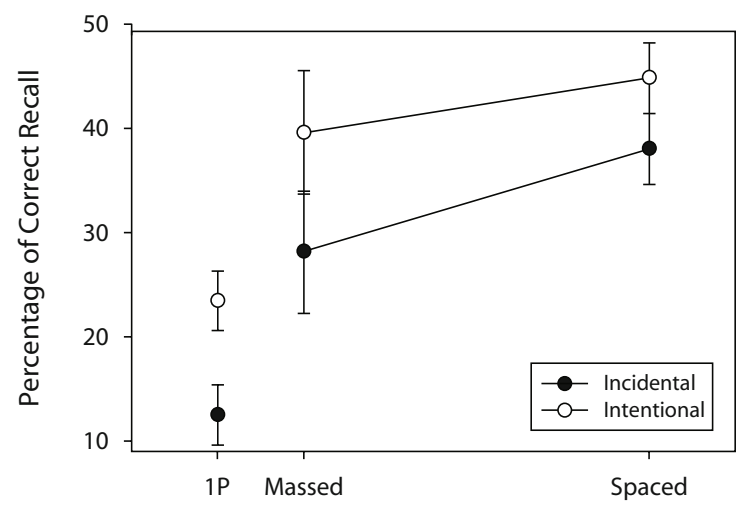

Repetition/Spacing

Figure 3. Mean percentage of correct free recall in Experiment 3 by college students, as a function of intentional versus incidental learning. 1P, once-presented items. Error bars represent 1 SEM. spacing effect depends on some aspect of processing that is limited in children and that changes with development. In Experiment 4, we will explore the nature of the limitation in children's processing and, by implication, what it is that changes as they grow into adults.

\section{EXPERIMENT 4}

Experimental findings on the spacing effect in children's free recall present an interesting puzzle. The fact that children exhibit a spacing effect in free recall under conditions of intentional learning but not under conditions of incidental learning is clear evidence that the mechanism producing the effect is based on a voluntary process. However, when learning is intentional, the evidence strongly supports the conclusion that the spacing effect in children is produced by an automatic, involuntary mechanism. Most notably, 3-year-olds yield a spacing effect in free recall, and the effect is not diminished when to-beremembered pictures are presented at a fast rate of $1 \mathrm{sec}$ per item (Toppino, 1991). How can these conflicting sets of findings be reconciled?

One hypothesis is that the spacing effect is produced by an automatic mechanism, but that the operation of this mechanism is contingent upon some aspect of intentional learning that acts like a switch. When the switch is on, the automatic process underlying the spacing effect is enabled. However, when the switch is off, as it apparently was in the incidental-learning conditions of our experiments, the spacing effect does not occur automatically.

The switch mechanism in children could be intentional learning per se. That is, early in development, the intent to learn may be necessary to activate the automatic process underlying the spacing effect. Alternatively, intentional learning may be correlated with some process that is sufficient to activate the automatic mechanism underlying the spacing effect, regardless of the intent to learn. A potential candidate in this regard is semantic processing.

Our incidental-learning procedures did not require semantic processing of the to-be-remembered pictures. With such a shallow processing task, the children in our experiments may have engaged in minimal semantic processing when learning was incidental, but engaged in more extensive or elaborate semantic processing when it was intentional. This would have produced the obtained pattern of results if minimal semantic processing was insufficient to enable an automatic spacing effect, but more extensive or elaborate semantic processing was sufficient.

Some encouragement for the semantic-processing hypothesis comes from recognition and frequency-judgment experiments that obtained a spacing effect with adults following incidental semantic processing of words, but not following incidental graphemic processing (Challis, 1993; Rose \& Rowe, 1976; see also Russo \& Mammarella, 2002). These findings have been interpreted as evidence for a deficient-processing account of the spacing effect, in which massed repetitions are remembered more poorly than spaced repetitions, because the former receive less semantic processing. For example, semantic processing of the second occurrence of a massed item may 
be minimized because it is highly primed by the first presentation (Challis, 1993), or because it is recognized as a repetition, and processing is terminated without the need for elaborate semantic processing (Rose, 1980; Rose \& Rowe, 1976). Although these hypotheses have been formulated in the context of research on cued-memory tasks involving conceptually driven processes (e.g., frequency judgments of meaningful stimuli), there is no obvious reason why similar processes could not influence other conceptually driven retrieval tasks, such as free recall.

In Experiment 4, we assessed the incidental free recall of fourth and fifth graders using a shallow processing task similar to that used in our previous studies versus a comparable semantic processing task. If the spacing effect in children is contingent upon the intent to learn per se, no spacing effect should be observed in either condition. However, if elaborate semantic-processing can enable an automatic spacing effect, children should exhibit a spacing effect when incidental learning involves a semantic processing task, but not when it involves a shallow processing task.

\section{Method}

Participants and Design. Participants were fourth- and fifthgrade children (mean age $=127.1$ months, range $=118-140$ months). The design was a $2 \times 3$ (type of processing $\times$ repetition/ spacing) mixed factorial. Type of processing (semantic vs. shallow) was varied between participants, and 24 children were randomly assigned to each condition. Repetition/spacing (once-presented, MP, DP) was varied within participants.

Materials. Lists of pictures were constructed in the same manner as for Experiments 1-3. In addition to half of the pictures appearing on each side of the screen, half of the pictures were of relatively small items, and half were of relatively large items. The actual pictures were all of a standard size, with small and large being differentiated according to whether their referent would or would not fit into the pocket of an apron that was used in the experiment. Across participants and lists, the items of each size occurred equally often on each side of the screen and within each repetition/spacing condition. One other change was that the screen was divided, so that the left half had a red background and the right half had a blue background.

Procedure. The procedure was the same as in Experiments 1-3, but with the following exceptions: In the semantic processing task, the children were required to predict whether the next picture would be of a small or a large item, defined by the relation of its referent to the pocket of the experimenter's apron. They responded by saying "small" or "large" before the picture was presented, and they could determine the accuracy of their prediction when the picture subsequently appeared. In this condition, however, evaluating the correctness of a prediction required semantic processing, because the actual size of the referent could not be determined from the size of the picture. In the shallow processing task, children predicted the side on which the next picture would appear, as in Experiments 1-3. However, so that both processing conditions would have a comparable verbal response, children in the shallow condition designated the predicted side of the screen by saying "red" or "blue." Pilot work had indicated that the distinction between left and right was confusing for some of the children.

\section{Results and Discussion}

Figure 4 presents the mean percentage of correct free recall, as a function of repetition/spacing and type of processing. The primary analysis was a $2 \times 2$ (type of processing $\times$ spacing) mixed ANOVA with repeated measures on the second factor. Semantic processing led to better recall

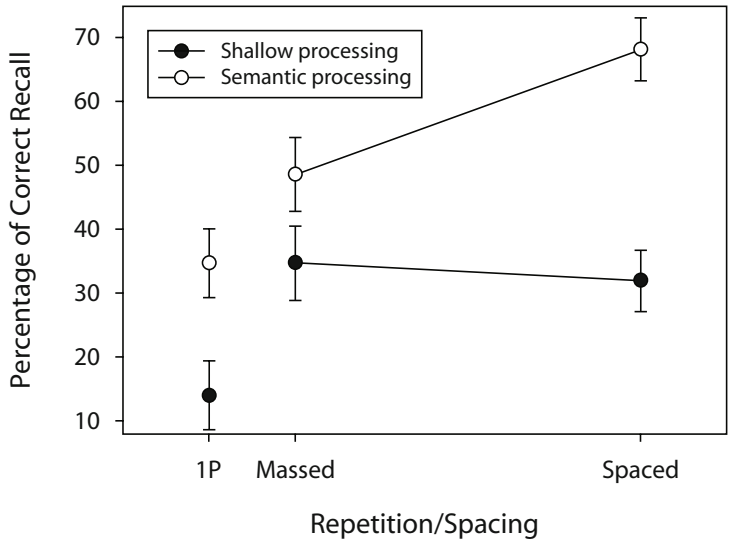

Figure 4. Mean percentage of correct incidental free recall by children in Experiment 4, as a function of semantic versus shallow processing. 1P, once-presented items. Error bars represent 1 SEM.

than did shallow processing $\left[F(1,46)=18.90, M S_{\mathrm{e}}=\right.$ 801.51]. Although the main effect of spacing was not reliable $[F(1,46)=2.91]$, there was a significant interaction involving spacing and the type of processing $[F(1,46)=$ 5.13, both $\left.M S_{\mathrm{e}} \mathrm{s}=583.35\right]$. The children exhibited a spacing effect following semantic processing $[t(47)=2.11]$ but not following shallow processing $[t(47)=0.67]$.

The second major analysis was a $2 \times 2$ mixed ANOVA with type of processing as the between-participants factor and repetition (once-presented vs. twice-presented) as the within-participants factor. Twice-presented items were recalled better than were once-presented items $[F(1,46)=$ $\left.16.81, M S_{\mathrm{e}}=665.24\right]$. Thus, once again, the failure of spacing to affect recall following shallow processing was obtained under conditions in which repetition did benefit recall. In addition, semantic processing yielded better recall than did shallow processing $\left[F(1,46)=29.22, M S_{\mathrm{e}}=\right.$ 432.93], but the type of processing did not interact with repetition $\left[F(1,46)<1.00, M S_{\mathrm{e}}=665.24\right]$.

The obtained differences in recall due to the type of processing in which the children engaged constitute the classic "levels-of-processing effect." The presence of this effect indicates that we were successful in inducing greater semantic processing by requiring the participants to predict whether a picture's referent would fit into the experimenter's apron pocket. The children in the shallowprocessing condition did not produce a spacing effect, replicating the results of Experiments 2 and 3. The spacing effect that was obtained in our semantic-processing condition indicates that the intent to learn is not necessary to enable an automatic spacing effect in children's free recall, and that engaging in relatively elaborate semantic processing is sufficient to do so.

\section{GENERAL DISCUSSION}

Our results both support and place limits on the hypothesis that the spacing effect in free recall is the product of 
an automatic, underlying mechanism. When learning was intentional, preschool children, elementary school children, and college students all exhibited a spacing effect. This persistent manifestation of the effect across an age span in which memory strategies range from minimal to highly sophisticated is consistent with the automaticity hypothesis. A limit to the automaticity of the spacing effect was revealed by the fact that the children exhibited a spacing effect in incidental free recall when they performed a task requiring relatively extensive or elaborate semantic processing, but not when they engaged in a shallow task requiring minimal semantic processing. This limitation on automaticity, however, was no longer apparent in young adults, who exhibited a spacing effect in incidental free recall after engaging in the same shallow processing task that had failed to yield a spacing effect in the children.

Nothing about elaborate semantic processing per se explains why free recall should improve with increased spacing between repetitions. Therefore, the fact that elaborate semantic processing led to a spacing effect in the children's incidental free recall suggests that its function was to enable a mechanism capable of automatically causing the effect. Put another way, the spacing effect in the children's incidental free recall was produced automatically, but the automatic mechanism did not operate unless the children voluntarily engaged in elaborate semantic processing.

Our findings clearly indicate that elaborate semantic processing is sufficient to enable an automatic spacing effect. Although we cannot rule out the extent of processing as the critical factor, semantic processing seems particularly important in the context of free recall, which may be the quintessential conceptually driven retrieval task. The semantic-processing hypothesis also accords well with other aspects of our data. First, participants of all ages may voluntarily engage in elaborate semantic processing when they know that their memory will be tested (e.g., Craik \& Tulving, 1975; Hyde \& Jenkins, 1969). This is consistent with the ubiquitous spacing effect obtained in intentionallearning conditions (Experiments 1-3). Second, as a result of extensive experience that younger children lack, college students may be more likely to process pictures semantically even when it is not explicitly required. This could occur either because relatively elaborate semantic processing has become automatized in college students, or because they are more likely to spontaneously adopt a strategy that incorporates elaborate semantic processing. Either alternative would account for the fact that, under shallow incidental-learning conditions, the college students exhibited a spacing effect (Experiment 3), whereas the children did not (Experiments 1,2, and 4).

If the spacing effect in our experiments is contingent upon the degree of semantic processing, what does this imply about the nature of the automatic mechanism that actually produces the effect? The most straightforward explanation of our findings is a deficient-processing account similar to that proposed in the context of cuedmemory research (Challis, 1993; Rose \& Rowe, 1976; Russo et al., 1998). When conceptually driven, explicit tests, such as free recall, are involved, performance is as- sumed to improve with increases in the total amount of semantic processing, accumulated across repetitions. As we noted earlier, Challis hypothesized that the amount of semantic processing was greater for spaced than for massed repetitions, as a result of automatic semantic priming of an item's second presentation. He proposed that the degree of priming declines with increased spacing between repetitions and is inversely related to the degree to which semantic information is elaborated on the second presentation. A similar mechanism was proposed by Rose and Rowe, who assumed that the second occurrence of a massed repetition would be recognized as a repetition, thus terminating processing after little semantic analysis. Either hypothesis would account for our results, as long as one assumes that the proposed mechanism would be rendered ineffective when learners fail to engage in elaborate semantic processing.

The fact that a theory developed in the context of cuedmemory research can account so well for our results involving children's free recall suggests that the spacing effect in cued-memory and free recall tasks may have more in common than recent trends in theorizing would suggest. However, although the theory accounts well for our data, it is limited by the fact that the proposed mechanisms involve short-lived processes. These mechanisms may contribute importantly to the markedly poor performance usually obtained with massed repetitions, relative to that obtained with repetitions involving short, distributed lags. However, the mechanisms seem inadequate, by themselves, to account for the facts that (1) performance often improves across a range of very long lags (e.g., Melton, 1970; Verkoeijen et al., 2005); (2) with longer lags, performance appears to be an inverted-U-shaped function of spacing (e.g., Toppino, Hara, \& Hackman, 2002; Verkoeijen et al., 2005); and (3) the optimal spacing between repetitions may increase with the length of the retention interval (e.g., Cepeda, Pashler, Vul, Wixted, \& Rohrer, 2006).

Contextual variability theories (without study-phase retrieval) do not easily explain why the presence or absence of the spacing effect depends on whether or not children engage in elaborate semantic processing. If contextual cues drift spontaneously with the passage of time and/or other events, and if they are encoded with an item automatically, a spacing effect should occur in incidental free recall, regardless of the level of processing. Even when contextual variability is embedded within a more comprehensive theory, such as Glenberg's (1979) component-levels theory, it is not clear why the effect of contextual variability would be insufficient to produce a spacing effect in the absence of relatively extensive semantic processing.

Theories that rely on study-phase retrieval may be more promising, because a learner's level of processing may affect the probability of successful study-phase retrieval. Elaborate semantic processing may be more likely than more shallow processing to yield successful study-phase retrieval, thus enabling the effective operation of contextual variability (Raaijmakers, 2003; Verkoeijen et al., 2004) or whatever other mechanism (e.g., retrieval practice; Thios \& D'Agostino, 1976) actually produces the 
spacing effect. However, this assumption by itself does not account for our results with children.

According to theories that incorporate study-phase retrieval, the effect of spacing repetitions is the result of two opposing forces (Toppino \& Bloom, 2002; Verkoeijen et al., 2005). The benefit of repetition, assuming successful study-phase retrieval, becomes greater as spacing increases (e.g., greater contextual variability, or more effective retrieval practice), whereas the likelihood of study-phase retrieval being successful declines as spacing increases. This leads to the expectation that performance will first improve and then decline as the spacing between repetitions becomes greater (e.g., Verkoeijen et al., 2005). The apex of this function should occur at a greater level of spacing when learning involves elaborate semantic processing, rather than more shallow processing, because the former is more favorable for successful study-phase retrieval. However, shallow processing should not eliminate the increasing portion of the spacing function; this part of the function should simply be restricted to shorter lags. Thus, a theory that includes study-phase retrieval does not necessarily predict our results with children, although it remains possible that such a theory could account for our results, with appropriate assumptions about the exact shape of the spacing function in the various processing conditions.

\section{Conclusion}

In agreement with the vast majority of studies that have investigated the spacing effect in free recall, our results support the hypothesis that the effect is produced by a mechanism that operates automatically. However, our results indicate that the automatic mechanism underlying the spacing effect is contingent on other processes that may be under voluntary control, and that, in children, the automatic mechanism can be enabled by elaborate semantic processing. It seems likely that this also applies to adults in whom elaborate semantic processing is more pervasive, perhaps because it has become automatized, or because adults are more likely to incorporate it into their current strategies. The role of elaborate semantic processing places constraints on the nature of the mechanism that actually produces the spacing effect in free recall. Our results seem to be accommodated most readily by a deficient-processing mechanism that moderates the amount of elaborate semantic processing received by a repeated item. An account involving study-phase retrieval (with or without contextual variability) also remains possible, but a hypothesis based on contextual variability without study-phase retrieval is not easily reconciled with our results.

\section{AUTHOR NOTE}

This research was supported in part by Grant HD21209 from the National Institute of Child Health and Human Development. The authors thank M. C. Klotz and Maura G. Sabatos for their assistance in collecting and analyzing the data for Experiments 2 and 4, respectively. We also thank three anonymous reviewers for their helpful comments on an earlier draft of this article. Correspondence concerning this article should be addressed to T. C. Toppino, Department of Psychology, Villanova
University, 800 Lancaster Ave., Villanova, PA 19085 (e-mail: thomas .toppino@villanova.edu).

\section{REFERENCES}

Baker-Ward, L., Ornstein, P. A., \& Holden, D. J. (1984). The expression of memorization in early childhood. Journal of Experimental Child Psychology, 37, 555-575.

Balota, D. A., DucheK, J. M., \& Paullin, R. (1989). Age-related differences in the impact of spacing, lag, and retention interval. Psychology \& Aging, 4, 3-9.

Braun, K., \& Rubin, D. C. (1998). The spacing effect depends on an encoding deficit, retrieval, and time in working memory: Evidence from once-presented words. Memory, 6, 37-65.

Cepeda, N. J., Pashler, H., Vul, E., Wixted, J. T., \& Rohrer, D. (2006). Distributed practice in verbal recall tasks: A review and quantitative synthesis. Psychological Bulletin, 132, 354-380.

Challis, B. H. (1993). Spacing effects on cued-memory tests depend on level of processing. Journal of Experimental Psychology: Learning, Memory, \& Cognition, 19, 389-396.

Craik, F. I. M., \& Tulving, E. (1975). Depth of processing and the retention of words in episodic memory. Journal of Experimental Psychology: General, 104, 268-294.

GLANZER, M. (1969). Distance between related words in free recall: Trace of the STS. Journal of Verbal Learning \& Verbal Behavior, 8 , 105-111.

Glenberg, A. M. (1979). Component-levels theory of the effects of spacing of repetitions on recall and recognition. Memory \& Cognition, 7, 95-112.

GreENE, R. L. (1989). Spacing effects in memory: Evidence for a twoprocess account. Journal of Experimental Psychology: Learning, Memory, \& Cognition, 15, 371-377.

HintzMAN, D. L. (1974). Theoretical implications of the spacing effect. In R. L. Solso (Ed.), Theories in cognitive psychology: The Loyola symposium (pp. 77-99). Hillsdale, NJ: Erlbaum.

Hintzman, D. L., \& Rogers, M. K. (1973). Spacing effects in picture memory. Memory \& Cognition, 1, 430-434.

Hyde, T. S., \& Jenkins, J. J. (1969). Differential effects of incidental tasks on the organization of recall of a list of highly associated words. Journal of Experimental Psychology, 82, 472-481.

Jensen, T. D., \& Freund, J. S. (1981). Persistence of the spacing effect in incidental free recall: The effect of external list comparisons and intertask correlations. Bulletin of the Psychonomic Society, 18, 183-186.

Kraft, R. N., \& Jenkins, J. J. (1981). The lag effect with aurally presented passages. Bulletin of the Psychonomic Society, 17, 132-134.

Mammarella, N., Avons, S. E., \& Russo, R. (2004). A short-term perceptual priming account of spacing effects in explicit cued-memory tasks for unfamiliar stimuli. European Journal of Cognitive Psychology, 16, 387-402.

Mammarella, N., Russo, R., \& Avons, S. E. (2002). Spacing effects in cued-memory tasks for unfamiliar faces and nonwords. Memory \& Cognition, 30, 1238-1251.

Melton, A. W. (1970). The situation with respect to the spacing of repetitions and memory. Journal of Verbal Learning \& Verbal Behavior, 9, 596-606.

Ornstein, P. A., Baker-WARD, L., \& Naus, M. J. (1988). The development of mnemonic skill. In F. E. Weinert \& M. Perlmutter (Eds.), Memory development: Universal changes and individual differences (pp. 31-50). Hillsdale, NJ: Erlbaum.

Pressley, M., \& Hilden, K. (2006). Cognitive strategies. In W. Damon \& R. M. Lerner (Series Eds.) \& D. Kuhn \& R. S. Siegler (Vol. Eds.), Handbook of child psychology: Vol 2. Cognition, perception, and language (6th ed., pp. 511-556). New York: Wiley.

RaAiJMaKers, J. G. W. (2003). Spacing and repetition effects in human memory: Application of the SAM model. Cognitive Science, 27, 431452.

Rea, C. P., \& Modigliani, V. (1987). The spacing effect in 4- to 9-yearold children. Memory \& Cognition, 15, 436-443.

Rose, R. J. (1980). Encoding variability, levels of processing, and the effects of spacing repetitions upon judgments of frequency. Memory \& Cognition, 8, 84-93. 
Rose, R. J., \& Rowe, E. J. (1976). Effects of orienting task and spacing of repetitions of frequency judgments. Journal of Experimental Psychology: Human Learning \& Memory, 2, 142-152.

Russo, R., \& Mammarella, N. (2002). Spacing effects in recognition memory: When meaning matters. European Journal of Cognitive Psychology, 14, 49-59.

Russo, R., PARkin, A. J., TAYlor, S. R., \& Wilks, J. (1998). Revising current two-process accounts of spacing effects in memory. Journal of Experimental Psychology: Learning, Memory, \& Cognition, 24, 161-172.

Schneider, W., \& BJorklund, D. F. (1998). Memory. In W. Damon (Series Ed.) \& D. Kuhn \& R. S. Siegler (Vol. Eds.), Handbook of child psychology: Vol 2. Cognition, perception, and language (5th ed., pp. 467-521). New York: Wiley.

Shaughnessy, J. J. (1976). Persistence of the spacing effect in free recall under varying incidental learning conditions. Memory \& Cognition, 4, 369-377.

Stoff, D. M., \& EAGLE, M. N. (1971). The relationship among reported strategies, presentation rate, and verbal ability and their effects on free recall learning. Journal of Experimental Psychology, 87, 423-428.

Thios, S., \& D'Agostino, P. R. (1976). Effects of repetition as a function of study-phase retrieval. Journal of Verbal Learning \& Verbal Behavior, 15, 529-537.

TopPINO, T. C. (1991). The spacing effect in young children's free recall: Support for automatic-process explanations. Memory \& Cognition, 19, 159-167.

ToppINo, T. C. (1993). The spacing effect in preschool children's free recall of pictures and words. Bulletin of the Psychonomic Society, 31, 27-30.

Toppino, T. C., \& Bloom, L. C. (2002). The spacing effect, free recall, and two-process theory: A closer look. Journal of Experimental Psychology: Learning, Memory, \& Cognition, 28, 437-444.
Toppino, T. C., \& DeMesquita, M. (1984). Effects of spacing repetitions on children's memory. Journal of Experimental Child Psychology, 37, 637-648.

Toppino, T. C., Hara, Y., \& Hackman, J. (2002). The spacing effect in the free recall of homogeneous lists: Present and accounted for. Memory \& Cognition, 30, 601-606.

UNDERWOOD, B. J. (1969). Some correlates of item repetition in freerecall learning. Journal of Verbal Learning \& Verbal Behavior, 8 , 83-94.

Underwood, B. J., KapelaK, S. M., \& Malmi, R. A. (1976). The spacing effect: Additions to the theoretical and empirical puzzles. Memory \& Cognition, 4, 391-400.

Van Strien, J. W., Verkoeijen, P. P. J. L., Van der Meer, N., \& Franken, I. H. A. (2007). Electrophysiological correlates of word repetition spacing: ERP and induced band power old/new effects with massed and spaced repetitions. International Journal of Psychophysiology, 66, 205-214.

Verkoeijen, P. P. J. L., Rikers, R. M. J. P., \& Schmidt, H. G. (2004). Detrimental influence of contextual change on spacing effects in free recall. Journal of Experimental Psychology: Learning, Memory, \& Cognition, 30, 796-800.

Verkoeijen, P. P. J. L., Rikers, R. M. J. P., \& Schmidt, H. G. (2005). Limitations to the spacing effect: Demonstration of an inverted u-shaped relationship between interrepetition spacing and free recall. Experimental Psychology, 52, 257-263.

Wilkinson, A. C., \& Koestler, R. (1983). Repeated recall: A new model and tests of its generality from childhood to old age. Journal of Experimental Psychology: General, 112, 423-451.

(Manuscript received August 26, 2008; revision accepted for publication November 11, 2008.) 